\title{
Tracheostomy reveals a rare aberrant right subclavian artery; a
}

\author{
case report \\ Neil K Chadha* and Sandro Chiti-Batelli
}

\author{
Address: Department of Otolaryngology - Head and Neck Surgery, St Mary's Hospital, London W2 1NY, United Kingdom \\ Email: Neil K Chadha* - neil_chadha@hotmail.com; Sandro Chiti-Batelli - sachb@tiscali.it \\ * Corresponding author
}

Published: 30 March 2004

BMC Ear, Nose and Throat Disorders 2004, 4:I

This article is available from: http://www.biomedcentral.com/l472-68/5/4/I

(C) 2004 Chadha and Chiti-Batelli; licensee BioMed Central Ltd. This is an Open Access article: verbatim copying and redistribution of this article are permitted in all media for any purpose, provided this notice is preserved along with the article's original URL.
Received: 22 September 2003

Accepted: 30 March 2004

\begin{abstract}
Background: Anomalies of vascular anatomy in the neck are well recognised. We present a case of a very rare aberrant artery discovered during tracheostomy.

Case presentation: Elective tracheostomy was performed in theatre for an elderly gentleman on long-term ventilation. Pre-operative examination of the neck revealed no obvious abnormality. During surgery, a large vessel was revealed overlying the tracheal fourth ring. This was found to be an anomalous right subclavian artery. The procedure was completed without complication.

Conclusions: The vessel abnormality described has not been previously documented in this context. It illustrates the importance of thorough pre-operative assessment of the neck and a sound knowledge of the potential for vascular abnormalities. The increasing prevalence of percutaneous dilatational tracheostomy techniques makes this lesson increasingly relevant.
\end{abstract}

\section{Background}

Anomalies of vascular anatomy in the neck are well recognised and described. Tracheostomy is a surgical procedure performed to gain direct access to the airway at the level of the upper trachea. This can be performed 'open' by incising and exposing the neck or 'closed' by percutaneous dilatational methods. We present a case of an unusual aberrant artery discovered during open tracheostomy. This emphasises the importance of an accurate physical examination prior to undertaking surgery, and illustrates the potential dangers of percutaneous tracheostomy.

\section{Case presentation}

An 82-year old gentleman presented with sub-acute large bowel obstruction. An emergency laparotomy revealed a perforated large bowel carcinoma with faecal peritonitis, and the patient was admitted to the Intensive Care Unit (ICU) after undergoing a hemicolectomy. He developed acute renal failure requiring haemo-filtration and remained intubated for ventilatory support. On day 15 post-operation, a tracheostomy was indicated to facilitate weaning from assisted ventilation. Local ICU policy prevented consideration of percutaneous tracheostomy, so an open tracheostomy was planned by the Ear, Nose and Throat surgeons. Examination of the neck revealed no obvious abnormality.

The tracheostomy proceeded in theatre under sterile conditions with a standard horizontal approach. The strap muscles were separated by blunt dissection in the vertical plane. It became clear at this stage that a large pulsatile vessel was overlying the trachea outside the pretracheal fascia. Meticulous and cautious dissection revealed the vessel to be running obliquely across the anterior surface of the trachea from left to right, at the level of the fourth tracheal ring (Figure 1). Further examination showed the 


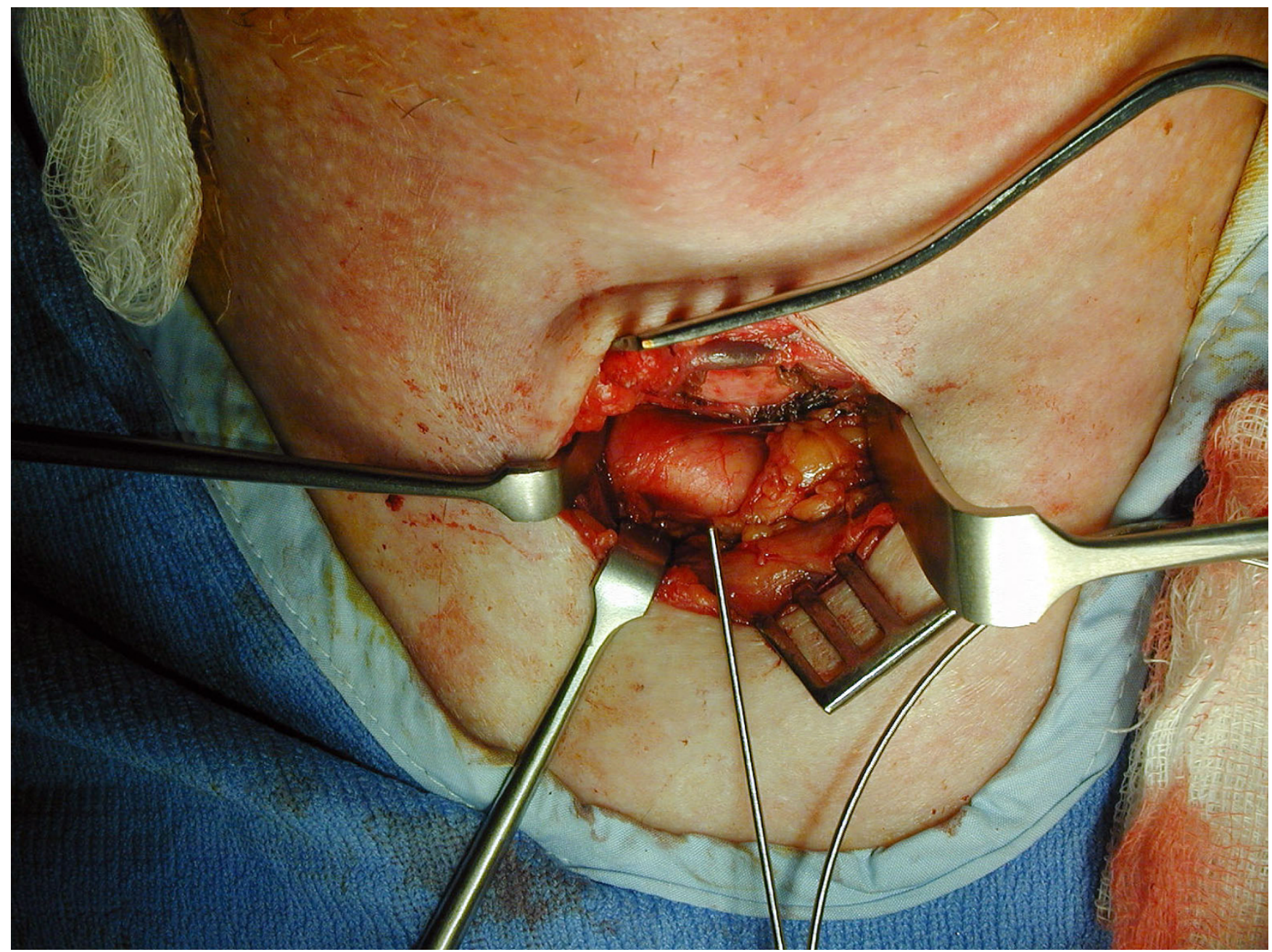

Figure I

Clinical photograph showing the aberrant right subclavian artery overlying the trachea.

artery to be an unusual aberrant right subclavian artery, arising directly from the aortic arch as the fourth major branch. The vessel appeared to gain its usual position by passing behind the right carotid after traversing the trachea.

A decision was made to continue with placement of the tracheostomy tube as it was felt this could be performed safely in the $2^{\text {nd }}$ to $3^{\text {rd }}$ rings. The risk of late erosion of the vessel by the tracheostomy tube was felt to be small. The thyroid isthmus was divided and fixed, and the trachea exposed from the cricothyroid inferiorly. A layer of fat and fascia was fashioned to lie superior to the aberrant subclavian artery to reduce the risk of contact with the tracheostomy tube. A window was made in the trachea at the $2^{\text {nd }}$ and $3^{\text {rd }}$ rings, and the tracheostomy placement continued without incident.

Post-operatively, the patient returned to the ICU department. He made good progress leading to decannulation 16 days later and discharge from ICU to a surgical ward. His further recovery on the ward was disrupted by the development of a severe bi-basal pneumonia and despite intensive treatment over the following weeks, he succumbed to overwhelming sepsis.

\section{Discussion}

The authors found no reported cases of this specific subclavian artery anomaly being discovered during surgery, although it is referred to in a standard anatomical textbook [1]. Anatomical variation of the aortic arch and great 
vessels are well recognised with an array of anomalies described [2-5]. Aberrant right subclavian artery is the most common abnormality of aortic arch development. In up to $0.5 \%$ of the population, the right subclavian arises as the fourth aortic branch, as in this case, but usually from the descending thoracic aorta [6]. Anomalies of this artery also include variations in its course (e.g. arteria lusoria passing dorsally between the oesophagus and spine).

The discovery of abnormal vasculature during percutaneous and open tracheostomy procedures has been previously described. Mukadam et al. (2002) recently reported a case of an aberrant brachio-cephalic artery discovered precluding a percutaneous dilatational tracheostomy [7]. This was palpated in the neck and confirmed by CT scan. The procedure was abandoned and an open tracheostomy was also felt to be too risky. Jarvis (1966) discovered a similar abnormality of the brachiocephalic artery during tracheostomy [8]. Nonetheless a tube was successfully placed in this case.

The discovery of anatomical variations of the aortic arch and its branches seems even more important in percutaneous dilatational tracheostomy (PDT) than open tracheostomy. PDT has gained acceptance in many centres as an alternative method to open surgical tracheostomy and was used in $78.4 \%$ of Intensive Care Units in the UK in 1998 [9]. Two methods of percutaneous tracheostomy are commonly employed, the guide-wire dilatational forceps technique, and the percutaneous dilatation technique first described by Ciaglia [10].

The advantages of percutaneous tracheostomy over open tracheostomy include its relative speed, simplicity, and the ability to perform it at the bedside. The major disadvantage is the increased risk of peri-operative complications including severe bleeding and damage to the posterior tracheal wall [11]. There are also relative contraindications to performing the percutaneous tracheostomy such as previous surgery, known anatomical abnormalities, and the obese neck. In a study of 497 cases of PDT, Muhammad et al. found $4.8 \%$ of procedures were abandoned due to bleeding [12]. The source of bleeding in four of these cases was attributed to variations in vascular anatomy. Interestingly, a case of fatality after PDT in a patient with aberrant right subclavian has previously been described [13].

\section{Conclusions}

This case illustrates the importance of awareness of the anatomical variability of the aortic arch branches while undertaking either open or percutaneous dilatational tracheostomy. In selected cases, a thorough neck examination can be augmented by ultrasound scan, Doppler studies, angiography or magnetic resonance scanning (in the non-emergency scenario). Therefore the rare but potentially severe risk of haemorrhage in the presence of anomalous vascular anatomy can be minimised if the surgeon maintains a high index of suspicion. In the presence of anatomical variations, open tracheostomy is safer than percutaneous dilatational tracheostomy and should be the procedure of choice.

\section{Acknowledgements}

Written consent was obtained from the patient or their relative for publication of the patient's photograph and details.

\section{References}

I. Williams PL: The subclavian artery. In Gray's Anatomy 38th edition. Churchill Livingstone, London; 1995:1530.

2. Bertolini A, Pelizza A, Panizzon G, Moretti R, Bava CL, Calza G, Tacchino A: Vascular rings and slings. Diagnosis and surgical treatment of 49 patients. I Cardiovasc Surg (Torino) 1987, 28:30I-I2.

3. Chun K, Colombani PM, Dudgeon DL, Aller JA Jr: Diagnosis and management of congenital vascular rings: 22 years experience. Ann Thorac Surg 1992, 53:597-02.

4. Fournial JF, Stanley P, Fouron JC, Guerin, Davignon A: [Abnormalities of the aortic arch. Apropos of 30 surgical cases in children]. Arch Mal Coeur Vaiss 1975, 68:485-95.

5. McElhinney DB, Thompson LD, Weinberg PM, Jue KL, Hanley FL: Surgical approach to complicated cervical aortic arch: anatomic, developmental, and surgical considerations. Cardiol Young 2000, 10(3):212-9.

6. Goldbloom AA: The anomalous right subclavian artery and its possible clinical significance. Surg Gynecol Obstet 1922, 34:378-84.

7. Mukadam GA, Hoskins E: Aberrant brachiocephalic artery precluding placement of tracheostomy. Anaesthesia 2002, 57:297-8.

8. Jarvis JF: Displaced brachiocephalic artery: a potential hazard in tracheostomy. S Afr Med J 1966, 40(17):396-7.

9. Cooper RM: Use and safety of percutaneous dilatational tracheostomy in intensive care. Anaesthesia 1998, 53:1209-27.

10. Ciaglia P, Firsching R, Syniec C: Elective percutaneous dilatational tracheostomy. A new simple bedside procedure; preliminary report. Chest 1985, 87(6):7I5-9.

II. Dulguerov P, Gysin C, Perneger TV, Chevrolet JC: Percutaneous or surgical tracheostomy: A meta-analysis. Crit Care Med 1999, 27:1617-1625.

12. Muhammad JK, Major E, Wood A, Patton DW: Percutaneous dilatational tracheostomy: haemorrhagic complications and the vascular anatomy of the anterior neck. A review based on 497 cases. Int J Oral Maxillofac Surg 2000, 29(3):217-22.

13. Shlugman D, Satya-Krishna R, Loh L: Acute fatal haemorrhage during percutaneous dilatational tracheostomy. $\mathrm{Br} J$ Anaesth 2003, 90(4):517-20.

\section{Pre-publication history}

The pre-publication history for this paper can be accessed here:

http://www.biomedcentral.com/1472-6815/4/1/prepub 